\title{
Emergency department and inpatient clinical decision tools for the management of febrile young infants among tertiary paediatric centres across Canada
}

\author{
Brett Burstein MD CM PhD MPH ${ }^{1,2, \oplus}$, Jocelyn Gravel MD MSc ${ }^{3, \oplus}$, Paul L. Aronson MD ${ }^{4}$, \\ Mark I. Neuman MD MPH ; on behalf of Pediatric Emergency Research Canada (PERC) \\ ${ }^{1}$ Division of Pediatric Emergency Medicine, Department of Pediatrics, Montreal Children's Hospital, McGill University \\ Health Centre, Montreal, Quebec; ${ }^{2}$ T.H. Chan School of Public Health, Harvard University, Boston, Massachusetts; \\ ${ }^{3}$ Division of Pediatric Emergency Medicine, Department of Pediatrics, Centre hospitalier universitaire Sainte-Justine, \\ Université de Montréal, Montreal, Quebec; ${ }^{4}$ Departments of Pediatrics and of Emergency Medicine, Section of Pediatric \\ Emergency Medicine, Yale-New Haven Children's Hospital, Yale School of Medicine, New Haven, Connecticut; ${ }^{5}$ Division \\ of Emergency Medicine, Department of Pediatrics, Boston Children's Hospital, Harvard Medical School, Boston, \\ Massachusetts
}

Correspondence: Brett Burstein, Montreal Children’s Hospital, McGill University Health Centre, 1001 Decarie Blvd, Montreal, Quebec H4C 3J1. Telephone 514-412-4400, fax 514-412-4397, e-mail brett.burstein@mail.mcgill.ca

\begin{abstract}
Objectives: With no nationally-endorsed guidelines and the emergence of newer diagnostic tools, there exists significant variation in the management of febrile infants $<90$ days. We sought to evaluate the prevalence and content of clinical decision tools (CDTs) for the emergency department (ED) and inpatient management of febrile young infants across Canada.

Methods: We undertook a cross-sectional analysis of febrile young infant CDTs from ED and inpatient units at all 16 Canadian tertiary paediatric hospitals. Additional data were collected using an electronic survey of ED and inpatient representatives, characterizing their clinical settings and diagnostic test availability. Content of all existent CDTs was independently reviewed using list items determined a priori. The primary outcome was the proportion of EDs and inpatient units with CDTs.

Results: Information regarding CDTs was gathered from all $16 \mathrm{EDs}$ and 16 inpatient units. CDTs were infrequently available $(9 / 32,28 \%)$, and were more common in the ED than inpatient setting $(8 / 16$ versus $1 / 16, \mathrm{P}=0.02)$. Review of existing CDTs revealed inter-centre differences for inclusion ages, treatment regimens, lumbar puncture recommendations, diagnostic testing and normal laboratory values. Despite availability reported at nearly all centres, C-reactive protein and respiratory virus testing were recommended in 3/9 and 5/9 CDTs, respectively. Procalcitonin testing was available at only $2 / 16(13 \%)$ centres, and not incorporated into any CDTs.

Conclusions: CDTs for the management of febrile young infants are infrequently available among Canadian tertiary paediatric centres, and rarely incorporate newer diagnostic tests. Heterogeneity among existent CDTs highlights the need for evidence-based unified ED and inpatient national guidelines.
\end{abstract}

Keywords: Fever; Guidelines; Sepsis; Septic workup; Serious bacterial infection.

Approximately $10 \%$ of febrile infants younger than 3 months of age harbour potentially life-threatening occult serious bacterial infections (SBIs) $(1,2)$. In the USA, several externally validated risk-stratification criteria have been developed for identifying 
febrile infants at low risk for SBI (3-5). These criteria have differing inclusion criteria based upon patient age, and provide conflicting recommendations for diagnostic testing, empiric antibiotic treatment and need for hospitalization. Importantly, these risk-stratification criteria also predate the availability of newer diagnostic tests, notably rapid respiratory virus testing $(6,7)$, C-reactive protein (CRP) $(8,9)$, and procalcitonin (8-10), each of which offer an enhanced ability to discriminate between low and high-risk infants.

In Canada, there are currently no nationally-endorsed guidelines for the management of febrile young infants, and no single protocol has been universally adopted. Consequently, there exists significant variation in care $(11,12)$. With the aim of balancing the risk of under-diagnosis and the potential harms of over-investigation, a majority of freestanding US paediatric hospitals have implemented institutional evidence-based clinical decision tools (CDTs) (13). CDTs incorporate best available evidence to improve quality of care, and clinical pathways, clinical practice guidelines and order sets are among the most commonly used CDTs (14-16). CDTs specific to the management of febrile young infants have been shown to reduce practice variation while optimizing clinical outcomes and costs (6).

The primary objective was to evaluate the prevalence of CDTs available to EDs and inpatient units at all 16 Canadian tertiary paediatric centres. Secondary objectives were to 1) compare the content of existing CDTs, and 2) determine the diagnostic tests currently utilized and readily available at each of the study sites. We hypothesized that febrile infant CDTs would be available at most hospitals, but that there would exist significant variability across sites.

\section{METHODS}

\section{Study design}

We performed a cross-sectional analysis of febrile young infant CDTs from EDs and inpatient units at each of the 16 Canadian tertiary paediatric hospitals. ED site representatives were identified using the email database of site representatives for the Pediatric Emergency Research Canada (PERC) network, a research consortium aimed at facilitating multicentre studies (17). ED site representatives assisted in identifying paediatricians who would be aware of inpatient guidelines from their institutions. Representatives were asked to provide all CDTs specific for the management of febrile young infants below 90 days available at their ED and inpatient units. Content of submitted CDTs was independently evaluated using a 10-item evaluation instrument of list items determined a priori. CDT evaluation items were generated from a previously published study assessing CDT use among US paediatric EDs (13). CDT content that was assessed included inclusion/exclusion criteria, recommended diagnostic testing, low-risk laboratory value definitions, indications for lumbar puncture and hospitalization, as well as suggested treatment regimens (drug and dosage). Existing CDTs were classified as a clinical pathway (protocol that standardizes management with detailed steps in an algorithm using time-frames or criteria-based progression), clinical practice guideline (framework that provides recommendations for diagnosis and management rather than specific protocols) or order set (predefined collections of medical orders that standardize treatment based on established guidelines) according to recognized definitions (14-16).

To obtain complementary data regarding site- and physician-level characteristics which could be associated with the presence of febrile infant CDTs, representatives from each unit were asked to complete a 14-question self-administered electronic survey between September and October of 2017 (www.limesurvey.org online platform, Hamburg, Germany). Previously published methods were employed for the survey design $(18,19)$ and dissemination $(20)$, which was developed in both English and French and piloted for clarity among 8 medical professionals not involved in the study. All survey fields were mandatory and required complete responses with no missing data. ED and inpatient representatives were asked multiple choice and open-ended questions to characterize their ED and inpatient settings as well as available diagnostic tests. For sites reporting existing $\mathrm{CDTs}$, respondents were asked questions regarding development, implementation and adherence. Participants were not surveyed regarding content of existing CDTs.

\section{Statistical analysis}

The primary outcome was the prevalence of CDTs in ED and in inpatient settings. We additionally compared the availability of CDTs between ED and inpatient settings, using a chi-squared test; a two-tailed P-value $<0.05$ was considered statistically significant. Descriptive and comparative analyses were performed using STATA v14 software (College Station, TX). The study was approved by the Research Ethics Board of the McGill University Health Centre and by the PERC Scientific Committee.

\section{RESULTS}

\section{ED and inpatient unit characteristics}

All 16 ED and inpatient study sites responded. Annual ED volume varied among centres with 11/16 (68.8\%) reporting $\geq 40,000$ patient visits/year (range $<10,000$ to $>80,000$ visits/ year). Ten of the 16 (62.5\%) EDs were staffed predominantly by physicians certified in Pediatric Emergency Medicine (PEM) by the Royal College of Physicians of Canada (RCPC). Among inpatient units, 10/16 (62.5\%) reported $\geq 40$ general paediatric medical beds, (range $<10$ to $>60$ ), and 
all were staffed predominantly by physicians RCPC-certified in Paediatrics. Five of 16 (31.3\%) EDs reported having dedicated short stay units in which eligible, nonadmitted febrile infants can be observed for $\geq 12$ hours, and 11/16 (68.8\%) reported access to a medical day hospital offering follow-up to discharged febrile infants. Respondents were asked about access for discharged febrile infants to next-day outpatient follow-up with primary healthcare providers; this was reported to be readily available to a majority of children on weekdays by only 6 of 32 of representatives (18.8\%) and on weekends by only 2 of $32(6.3 \%)$.

\section{CDT prevalence across Canadian tertiary paediatric hospitals}

Overall, CDTs of any type were available among 9 of 32 units $(28.1 \%)$ at 8 of $16(50.0 \%)$ hospitals. CDTs were more often available in the $\operatorname{ED}(8 / 16,50.0 \%)$ than the inpatient setting (1/16, 6.3\%, $\mathrm{P}=0.02)$; one site had both $\mathrm{ED}$ and inpatient CDTs. By CDT type, clinical pathways, practice guidelines and order sets were available at 5/32 (15.6\%), 2/32 (6.2\%), and $4 / 32(12.5 \%)$ units, respectively. ED CDTs were available at $1 / 5(20.0 \%), 3 / 5(60.0 \%)$, and $4 / 6(66.7 \%)$ of centres with $<40,000,40-<70,000$, and $\geq 70,000$ annual visits, respectively. Similarly CDTs were present in 2/6 (33.3\%), 3/6 (50.0\%), and 4/6 (66.7\%) EDs with $<50 \%, 50-<80 \%$, and $\geq 80 \%$ PEMtrained physicians.

\section{CDT development and implementation processes}

Nearly all CDTs $(8 / 9,88.9 \%)$ were implemented after 2002, and all but one have been updated more recently than 2012 . Few reported tracking of provider adherence or audits of impact $(3 / 9,33 \%)$. All eight of the ED CDTs were developed locally by PEM physicians in consultation most frequently with infectious disease specialists (6/8, 75\%; Figure 1A). Few ED CDTs were developed in consultation with inpatient physicians $(3 / 8,37.5 \%)$. Strategies most commonly employed to implement CDTs in the ED were the circulation of materials to Department members and creation of Internet-based reference documents (both 5/8, 62.5\%; Figure 1B).

\section{Content of existing CDTs}

All nine units with existing CDTs submitted these for independent content review and comparative summaries are available in online-only materials. Review of existing CDTs revealed inter-centre differences for inclusion ages, exclusion criteria, and high-risk clinical features and diagnostic testing recommendations (Table 1). All CDTs defined fever as a rectal temperature $\geq 38.0^{\circ} \mathrm{C}$. Hospitalization and empiric parenteral antibiotic

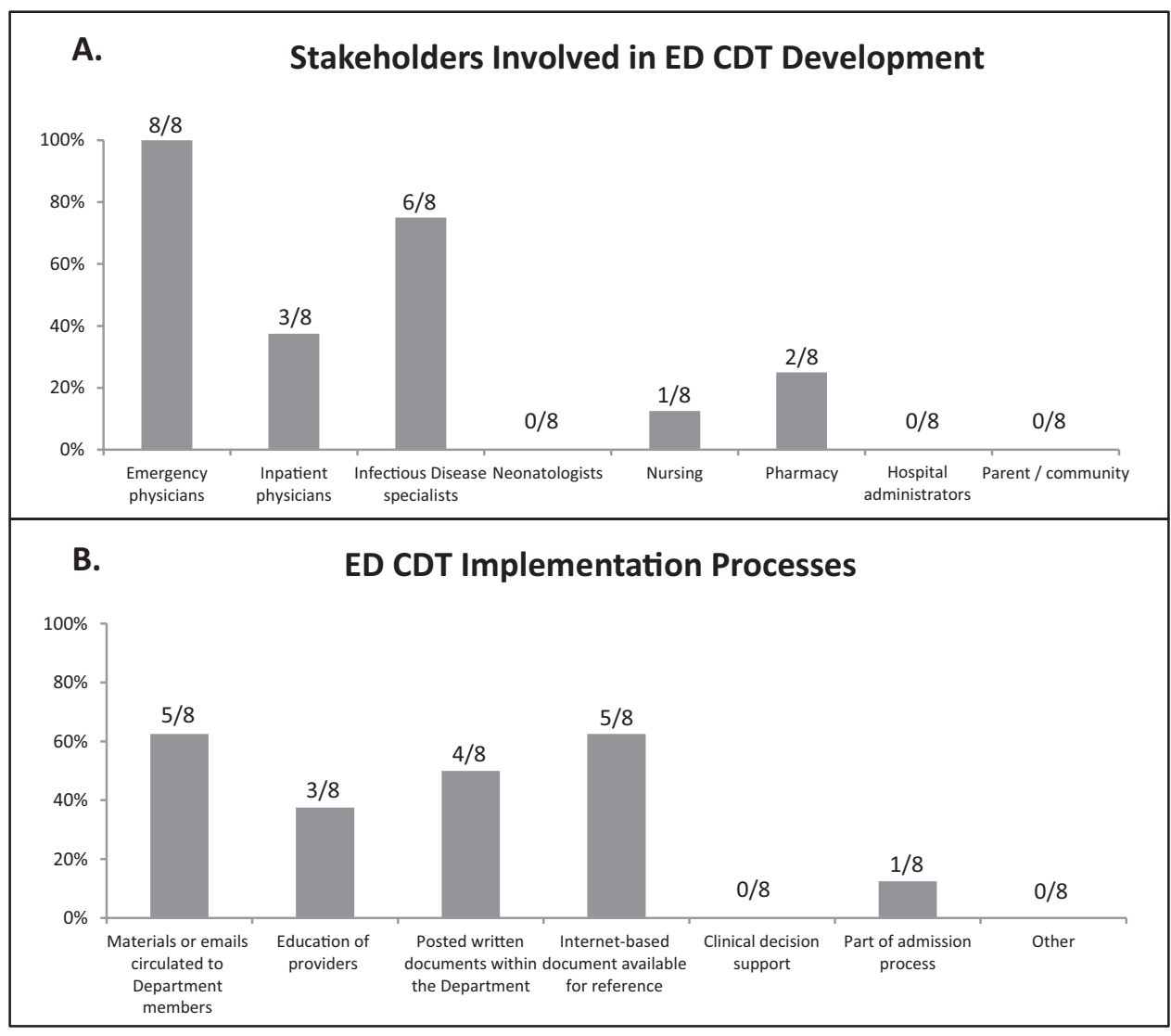

Figure 1. Clinical decision tool development (upper panel, A) and implementation processes (lower panel, B). 


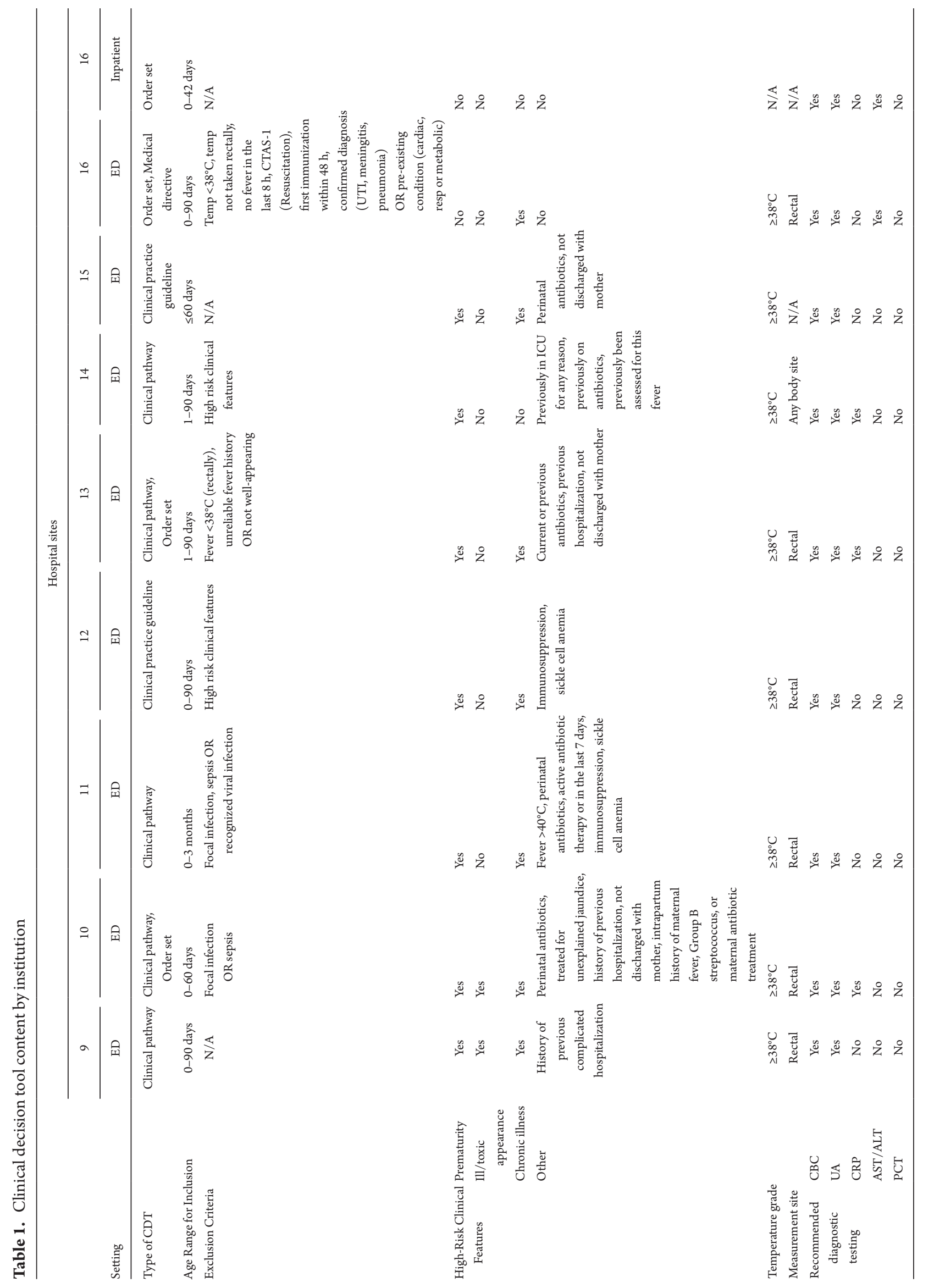




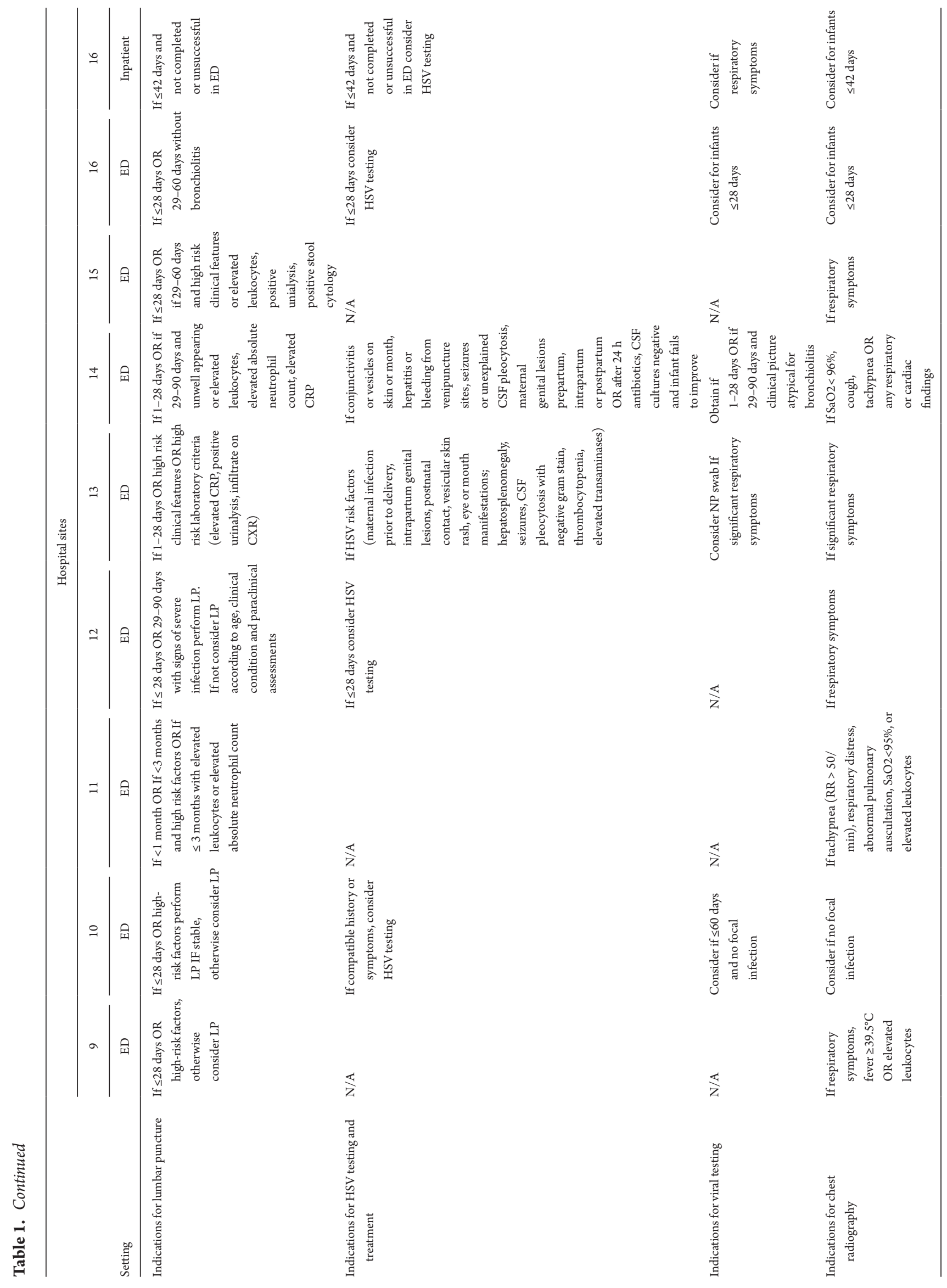




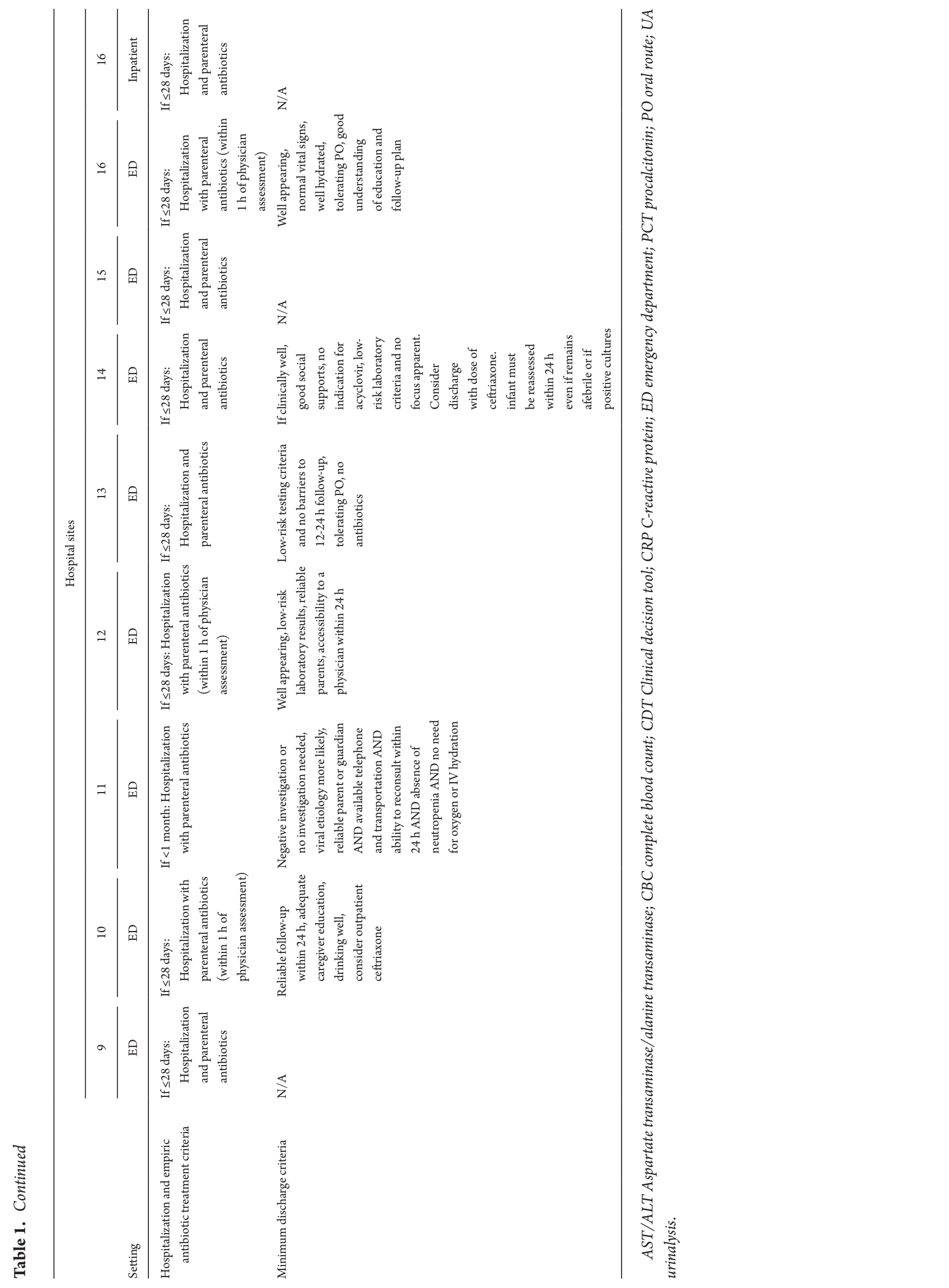


treatment was recommended for infants $\leq 28$ days at all sites. Only two CDTs suggested consideration of outpatient management with intravenous antibiotic coverage (ceftriaxone) for infants $>28$ days. Discharge criteria were similar among CDTs with 5/9 (55.6\%) specifying that follow-up within 24 hours should be ensured. Antibiotic treatment regimens differed between sites (Table 2).

\section{Diagnostic test availability and CDT recommended testing}

Urinalysis and complete blood count ( $\mathrm{CBC}$ ) testing was recommended in all CDTs (Table 3). Despite availability reported at all centres, CRP was rarely incorporated into existent CDTs $(3 / 9,33.3 \%)$. Multiplex respiratory virus testing was included among 5/9 (55.6\%) of CDTs, but indication varied by institution depending on patient age and presence of respiratory symptoms. Five of nine CDTs (55.6\%) address cerebrospinal fluid (CSF) testing for herpes simplex virus infection (HSV), though with differing recommendations. Liver transaminases, imperfect but potentially important markers for disseminated HSV $(6,21,22)$, were never included among existing CDTs. Procalcitonin, the laboratory test with highest sensitivity and specificity for detection of SBI in febrile young infants $(8,10)$, was reported available at only $2 / 16(12.5 \%)$ centres, and was not incorporated into any CDTs. CSF testing, which is universally available, was recommended in all CDTs for infants in the first month. Most CDTs $(7 / 9,77.8 \%)$ did not endorse routine lumbar puncture for infants beyond 28 days, however indications and ages for lumbar puncture varied for this older age group between centres, and between ED and inpatient CDTs at the same site. Low-risk laboratory values to guide management were inconsistently included among CDTs, and frequently differed between centres when specified.

\section{DISCUSSION}

This cross-sectional analysis of all Canadian tertiary paediatric ED and inpatient units revealed that CDTs for the management of febrile young infants are more frequent in the ED setting, but not widely available overall. Among existent CDTs, there exists considerable inter-institution variation, which differ by inclusion criteria, high-risk clinical and laboratory findings, diagnostic testing, antibiotic treatment regimens, and indications for lumbar puncture. When present, existent CDTs rarely incorporate information on newer, higher performing diagnostic tests, such as procalcitonin (8-10).

The overall paucity of institutional CDTs nationwide was counter to the a priori hypothesis of this study. In Canada, evidence for management of febrile young infants was last examined by the Canadian Paediatric Society in 1996 and excluded neonates (23), and there are currently no nationally-endorsed guidelines. A 2009 study from six Canadian tertiary paediatric EDs demonstrated significant practice variation for febrile young infants, particularly in terms of CSF testing and antibiotic treatment (11). Few existing CDTs, compounded with heterogeneity of those that exist, may in part underlie ongoing practice variation. A study of 37 freestanding US children's hospitals in 2014 similarly found substantial patient- and hospital-level variation in the management of febrile young infants (2). Twenty-one of 33 of these centres (64\%) reported institutional clinical practice guidelines, and while they show relative uniformity in recommendations for infants under 1 month, there is significant heterogeneity for the management of older infants (13). In a systematic assessment of all 25 hospitals providing paediatric care in Israel in 2014, only 9 (36\%) were found to have written protocols regarding febrile young infants, and significant differences were noted among those in use (24).

Clinical practice guidelines can improve healthcare quality and outcomes while reducing utilization of low-value clinical services (25). Implementation of CDTs for febrile young infants has been shown to reduce the time to diagnostic test collection (26), time to antibiotic administration $(26,27)$, decrease practice variation, and ensure appropriate testing (6). A set of febrile young infant CDTs implemented across a healthcare system in Utah decreased variability with excellent clinical outcomes and reduced costs (6). Importantly, the presence of CDTs does not ensure knowledge of, or adherence to guidelines (28).

In the current context of widespread immunizations and antenatal Group B streptococcus screening/prophylaxis, there has been a shift in SBI epidemiology among febrile young infants $(29,30)$. There is broad consensus on the need for new evidence-based guidelines for the management of febrile young infants (31). Important work remains toward establishing updated national guidelines incorporating newer diagnostic tests (9,32). At present, the highest performing laboratory tests are infrequently incorporated into Canadian CDTs and procalcitonin testing is not widely available. There was a trend towards CDTs existing at higher volume centres with more PEM-trained physicians. CDTs would likely be of particular benefit in smaller, nontertiary and nonpaediatric centres where febrile young infants may be managed less frequently. We have provided summaries of existing CDTs that centres can use to compare or as a starting point for development.

The present study has limitations. We solicited participation from the site representatives most appropriate to comment on febrile young infant management, though it possible that these representatives did not have perfect knowledge of the CDTs in place. As such, we cannot completely exclude the possibility of misclassification resulting from answers provided by site representatives regarding CDT implementation processes. However, we collected CDTs when existent for independent analysis to minimize recall bias of CDT content. Our primary outcomes 


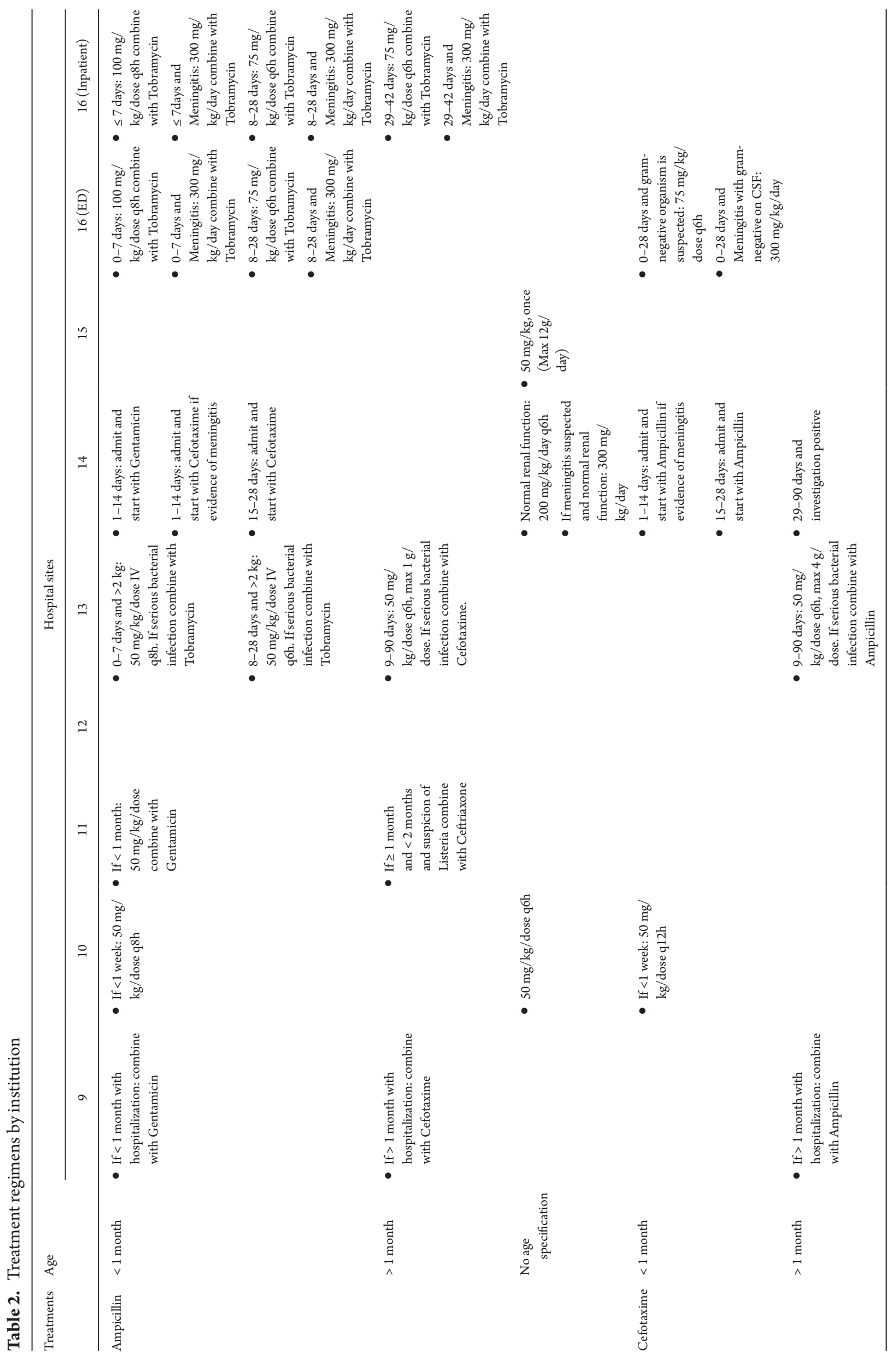




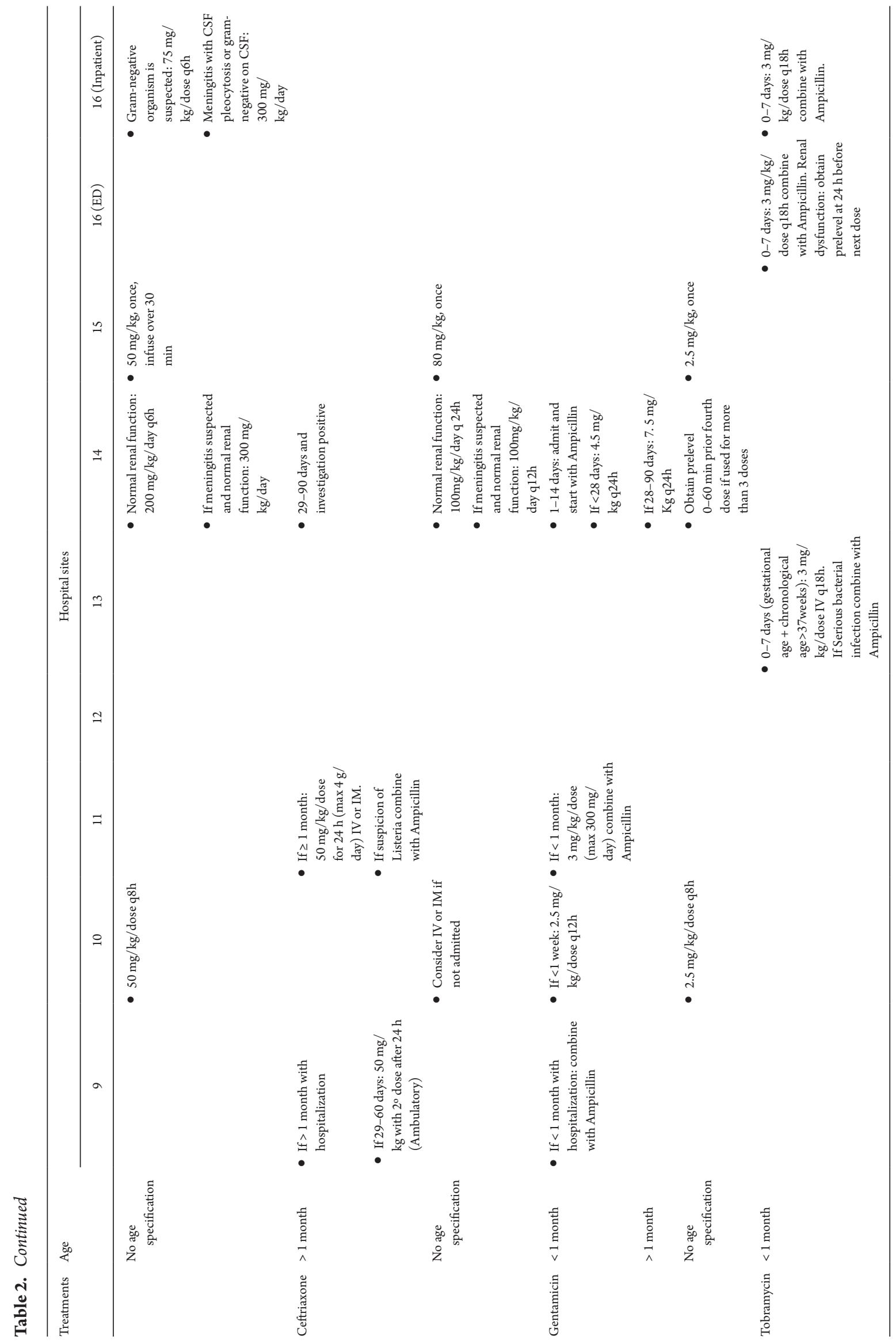




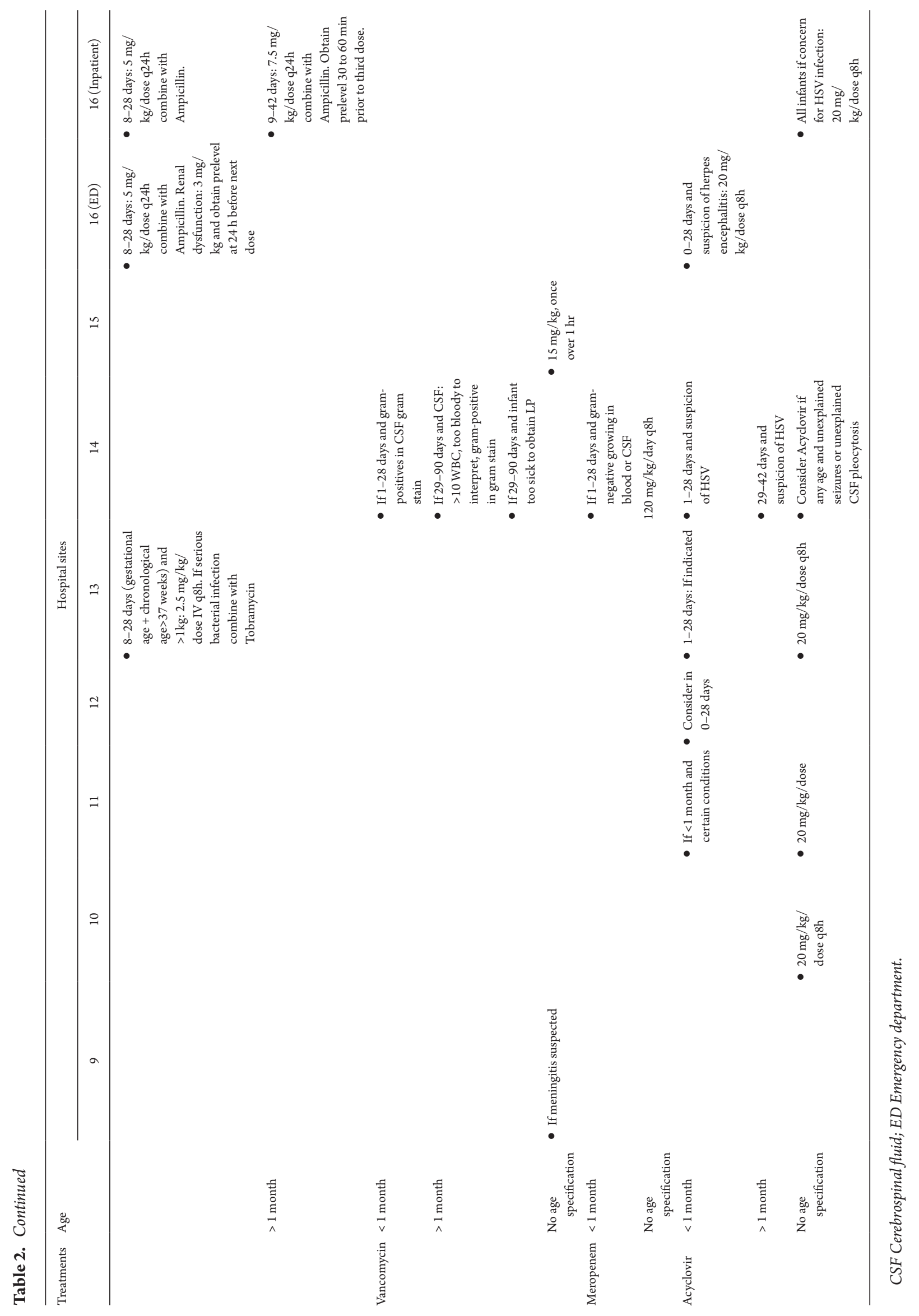




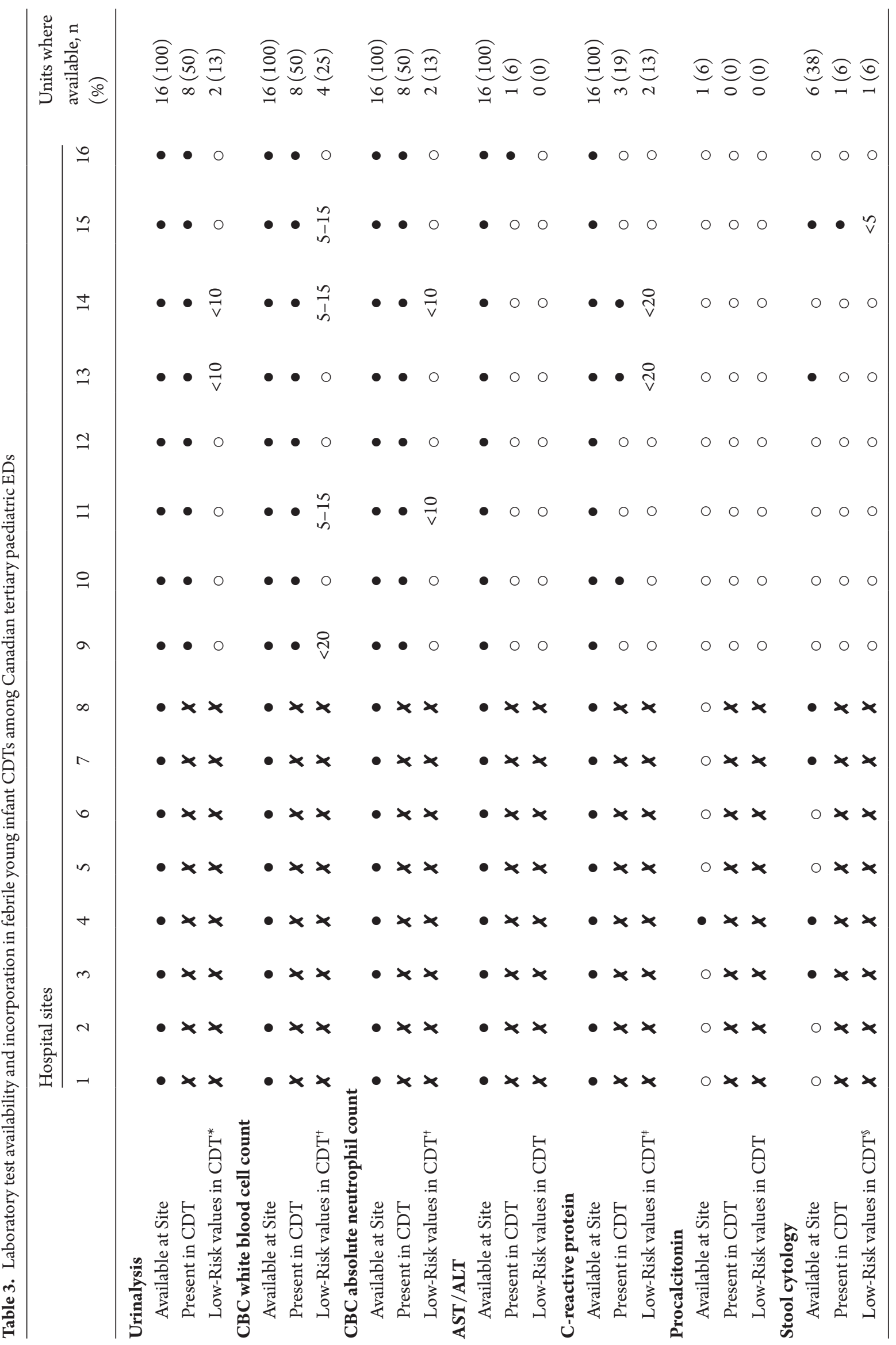




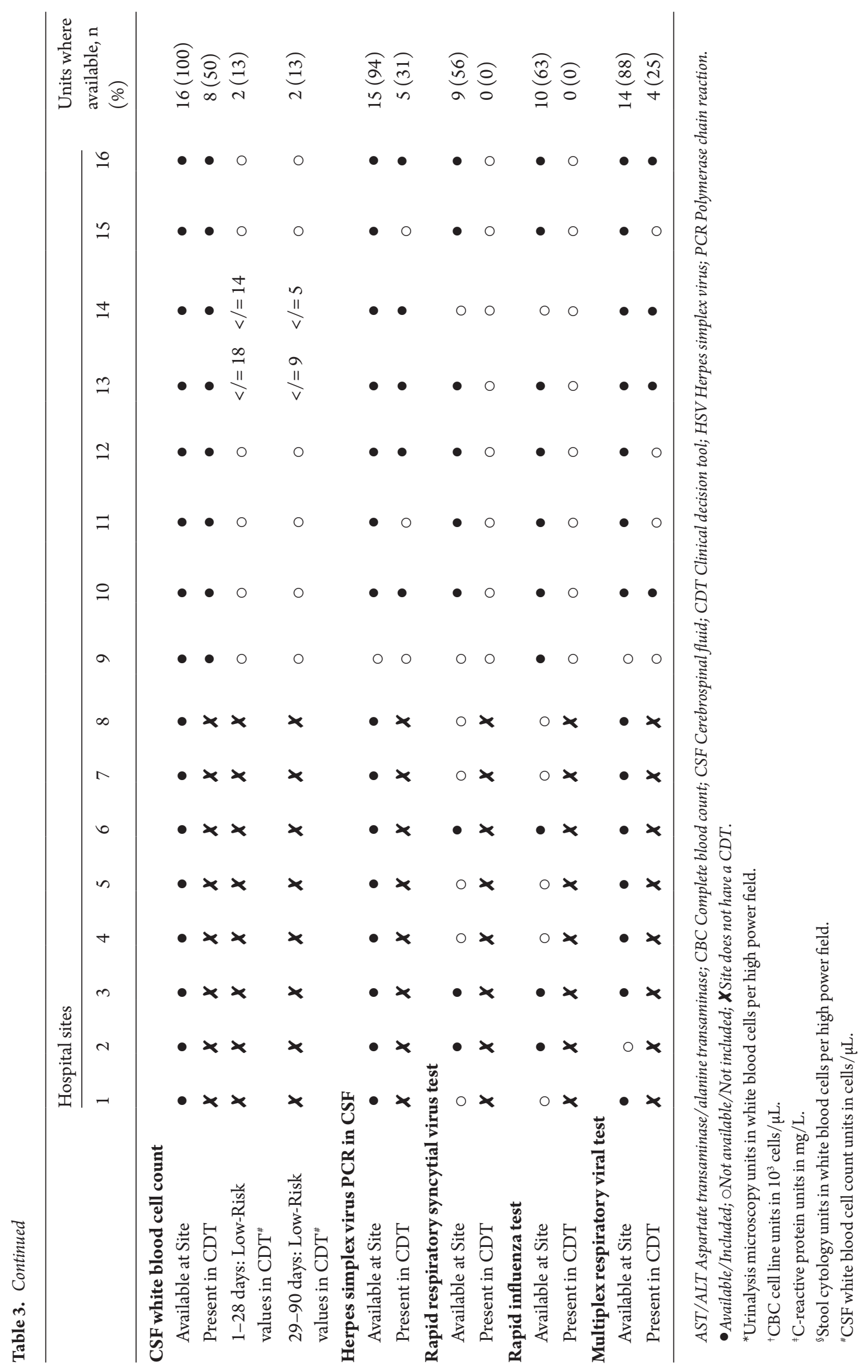


of interest were the prevalence and content of existing CDTs, and we did not assess adherence, nor clinical outcomes with the differing CDTs. We report on the CDTs in place in the ED and inpatient units of tertiary paediatric centres only, and CDTs presented may not be generalizable to nontertiary or nonpaediatric institutions.

\section{CONCLUSIONS}

CDTs designed to standardize the care of febrile young infants are not frequent among Canadian tertiary paediatric centres. Variation among existing CDTs highlights the need for the establishment of national practice guidelines. With data emerging from more recent studies using novel risk-stratification strategies, there is a significant need for updated evidence-based recommendations to inform institutional CDTs. A unified ED and inpatient CDT could help minimize practice variation, improve patient-centred outcomes, and inform future well designed prospective studies.

\section{Acknowledgements}

The authors thank Raphael Freitas (McGill University Health Centre) for technical assistance, and Dr. Jess Papenburg for thoughtful revision of the manuscript (Montreal Children's Hospital, Division of Pediatric Infectious Diseases).

Financial Disclosures: The authors have no financial relationships to disclose

Funding Source: PLA is supported by Clinical and Translational Science Awards grant KL2 TR001862 from the National Center for Advancing Translational Science, a component of the National Institutes of Health. The content is solely the responsibility of the authors and does not necessarily represent the official views of the National Institutes of Health.

Potential Conflicts of Interest: No reported conflicts of interest. All authors have submitted the ICMJE Form for Disclosure of Potential Conflicts of Interest. Conflicts that the editors consider relevant to the content of the manuscript have been disclosed.

\section{References}

1. Baraff LJ, Bass JW, Fleisher GR, et al. Practice guideline for the management of infants and children 0 to 36 months of age with fever without source. Agency for health care policy and research. Ann Emerg Med 1993;22(7):1198-210.

2. Aronson PL, Thurm C, Alpern ER, et al.; Febrile Young Infant Research Collaborative. Variation in care of the febrile young infant $<90$ days in US pediatric emergency departments. Pediatrics 2014;134(4):667-77.

3. Baskin MN, O'Rourke EJ, Fleisher GR. Outpatient treatment of febrile infants 28 to 89 days of age with intramuscular administration of ceftriaxone. J Pediatr 1992;120(1):22-7.

4. Baker MD, Bell LM, Avner JR. Outpatient management without antibiotics of fever in selected infants. N Engl J Med 1993;329(20):1437-41.

5. Jaskiewicz JA, McCarthy CA, Richardson AC, et al. Febrile infants at low risk for serious bacterial infection-an appraisal of the Rochester criteria and implications for management. Febrile infant collaborative study group. Pediatrics 1994;94(3):390-6
6. Byington CL, Reynolds CC, Korgenski $\mathrm{K}$, et al. Costs and infant outcomes after implementation of a care process model for febrile infants. Pediatrics 2012;130(1):e16-24

7. Byington CL, Enriquez FR, Hoff C, et al. Serious bacterial infections in febrile infants 1 to 90 days old with and without viral infections. Pediatrics 2004;113(6):1662-6.

8. Yo $\mathrm{CH}, \mathrm{Hsieh} \mathrm{PS}$, Lee $\mathrm{SH}$, et al. Comparison of the test characteristics of procalcitonin to C-reactive protein and leukocytosis for the detection of serious bacterial infections in children presenting with fever without source: a systematic review and meta-analysis. Ann Emerg Med 2012;60(5):591-600.

9. Hui C, Neto G, Tsertsvadze A, et al. Diagnosis and management of febrile infants (0-3 months). Evid Rep Technol Assess (Full Rep) 2012;(205):1-297.

10. Milcent K, Faesch S, Gras-Le Guen C, et al. Use of procalcitonin assays to predict serious bacterial infection in young febrile infants. JAMA Pediatr 2016;170(1):62-9.

11. Goldman RD, Scolnik D, Chauvin-Kimoff L, et al.; Fever in Infants Group Research, Pediatric Emergency Research of Canada. Practice variations in the treatment of febrile infants among pediatric emergency physicians. Pediatrics 2009;124(2):439-45.

12. Burstein B, Dubrovsky AS, Greene AW, Quach C. National survey on the impact of viral testing for the ED and inpatient management of febrile young infants. Hospital Pediatrics 2016;6(4):226-33.

13. Aronson PL, Thurm C, Williams DJ, et al.; Febrile Young Infant Research Collaborative. Association of clinical practice guidelines with emergency department management of febrile infants $\leq 56$ days of age. J Hosp Med 2015;10(6):358-65.

14. Bergman DA. Evidence-based guidelines and critical pathways for quality improvement. Pediatrics 1999;103 (1 Suppl E):225-32.

15. Lawal AK, Rotter T, Kinsman L, et al. What is a clinical pathway? Refinement of an operational definition to identify clinical pathway studies for a Cochrane systematic review. BMC Med 2016;14:35.

16. U.S. National Library of Medicine. Medical Subject Headings (March 1, 2017) <http://www.nlm.nih.gov/ mesh/>. Accessed March 1, 2017.

17. Bialy L, Plint A, Zemek R, et al.; Pediatric Emergency Research Canada (PERC). Pediatric emergency research Canada: Origins and evolution. Pediatr Emerg Care 2018;34(2):138-44.

18. Burns KE, Duffett M, Kho ME, et al.; ACCADEMY Group. A guide for the design and conduct of self-administered surveys of clinicians. CMAJ 2008;179(3):245-52.

19. Trottier ED, Ali S, Le May S, Gravel J. Treating and reducing anxiety and pain in the paediatric emergency department: The TRAPPED survey. Paediatr Child Health 2015;20(5):239-44.

20. Dillman DA. Mail and Internet Surveys: The Tailored Design Method, 2nd edn Hoboken, NJ: John Wiley \& Sons, 2000.

21. McGoogan KE, Haafiz AB, González Peralta RP. Herpes simplex virus hepatitis in infants: Clinical outcomes and correlates of disease severity. J Pediatr 2011;159(4):608-11

22. Curfman AL, Glissmeyer EW, Ahmad FA, et al. Initial presentation of neonatal herpes simplex virus infection. J Pediatr 2016;172:121-126.e1.

23. Infectious Diseases and Immunization Committee CPS. Management of the febrile one- to 36-month-old child with no focus of infection. Paediatr Child Health 1996;1:41-5.

24. Yarden-Bilavsky H, Ashkenazi S, Amir J, Schlesinger Y, Bilavsky E. Fever survey highlights significant variations in how infants aged $\leq 60$ days are evaluated and underline the need for guidelines. Acta Paediatr 2014;103(4):379-85.

25. Institute of Medicine US. Committee on standards for developing trustworthy clinical practice guidelines. In: Graham R, Mancher M, Wolman DM, Sheldon Greenfiel S, Steinberg E, eds. Clinical Practice Guidelines We Can Trust. Washington, DC National Academies Press, 2011.

26. Murray AL, Alpern E, Lavelle J, Mollen C. Clinical pathway effectiveness: febrile young infant clinical pathway in a pediatric emergency department. Pediatr Emerg Care 2017;33(9):e33-7.

27. Sharieff GQ, Hoecker C, Silva PD. Effects of a pediatric emergency department febrile infant protocol on time to antibiotic therapy. J Emerg Med 2001;21(1):1-6.

28. Meehan WP III, Fleegler E, Bachur RG. Adherence to guidelines for managing the well-appearing febrile infant: Assessment using a case-based, interactive survey. Pediatr Emerg Care 2010;26(12):875-80.

29. Biondi E, Evans R, Mischler M, et al. Epidemiology of bacteremia in febrile infants in the United States. Pediatrics 2013;132(6):990-6.

30. Greenhow TL, Hung YY, Herz AM, Losada E, Pantell RH. The changing epidemiology of serious bacterial infections in young infants. Pediatr Infect Dis J 2014;33(6):595-9.

31. Schneider C, Blumberg S, Crain EF. A survey of the management of febrile infants in pediatric emergency departments. Pediatr Emerg Care 2012;28(10):1022-6.

32. Woll C, Neuman MI, Aronson PL. Management of the febrile young infant: Update for the $21^{\text {st }}$ century. Pediatr Emerg Care 2017;33(11):748-53. 